\title{
Optogenetic control of the Bicoid morphogen reveals fast and slow modes of gap gene regulation
}

Anand P. Singh ${ }^{1 *}$, Ping Wu ${ }^{2 *}$, Sergey Ryabichko ${ }^{1}$, João Raimundo ${ }^{1}$, Michael Swan ${ }^{1,2}$, Eric Wieschaus ${ }^{1,2, * *}$, Thomas Gregor ${ }^{1,3 * *}$, Jared E. Toettcher ${ }^{2, * *}$

${ }^{1}$ Lewis Sigler Institute for Integrative Genomics

Princeton University, Princeton NJ 08544

${ }^{2}$ Department of Molecular Biology

Princeton University, Princeton NJ 08544

${ }^{3}$ Department of Physics

Princeton University, Princeton NJ 08544

* Contributed equally

** Co-corresponding authors

Correspondence should be addressed to:

Jared E Toettcher

toettcher@princeton.edu

Thomas Gregor

tg2@princeton.edu

Eric F Wieschaus

efw@princeton.edu 


\section{Abstract}

2 Developmental patterning networks are regulated by multiple inputs and feedback connections

3 that rapidly reshape gene expression, limiting the information that can be gained solely from

4 slow genetic perturbations. Here we show that fast optogenetic stimuli, real-time transcriptional

5 reporters, and a simplified genetic background can be combined to reveal quantitative regulatory

6 dynamics from a complex genetic network in vivo. We engineer light-controlled variants of the

7 Bicoid transcription factor and study their effects on downstream gap genes in embryos. Our

8 results recapitulate known relationships, including rapid Bicoid-dependent expression of giant

9 and hunchback and delayed repression of Krüppel. In contrast, we find that the posterior pattern

10 of knirps exhibits a quick but inverted response to Bicoid perturbation, suggesting a previously

11 unreported role for Bicoid in suppressing knirps expression. Acute modulation of transcription

12 factor concentration while simultaneously recording output gene activity represents a powerful

13 approach for studying how gene circuit elements are coupled to cell identification and complex

14 body pattern formation in vivo. 


\section{Main Text}

16 Gene networks play a crucial role in developmental patterning, transforming rudimentary

17 positional cues into a multitude of sharply defined domains of gene expression. Such networks

18 are typically characterized by redundant inputs to ensure that gene expression is initialized

19 appropriately, and feedback connections between genes in the network to ensure a consistent

20 patterning response. Information from these inputs is integrated at enhancers that bind multiple

21 transcription factors and control gene expression through transient interactions with promoters

22 and longer-term alterations of chromatin structure and accessibility. Understanding how

23 networks function requires knowing the time scales over which individual components operate.

The gap gene network of the early Drosophila embryo is a canonical example of such a

26 sophisticated pattern-forming system. In this network, the expression of four core transcription

27 factors - the gap genes giant (gt), hunchback (hb), Krüppel (Kr), and knirps (kni) - is initiated by

28 three partially-redundant sources of positional information that are maternally deposited in the

29 egg. These maternally supplied inputs include an anterior-to-posterior gradient of the Bicoid

30 (Bcd) transcription factor, a posterior-to-anterior gradient of the Nanos RNA binding protein,

31 and Torso receptor tyrosine kinase signaling at the anterior and posterior poles. In addition to

32 responding to maternal inputs, the four gap genes further regulate themselves and each other to

33 generate bands of gene expression that are essential for specifying the body plan (Figure 1a-b) ${ }^{1}$.

35 A useful first step in disentangling such networks has been to characterize transcriptional

36 responses under conditions where input information has been reduced to single components or

37 flattened so that all cells in the embryo see the same input values (Figure 1c-d). For example, 
a

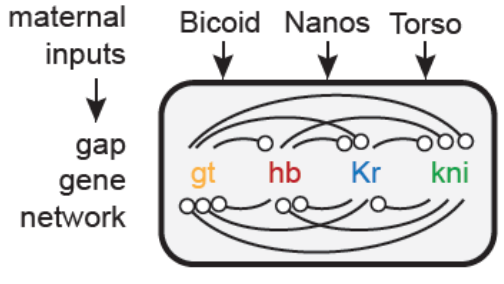

$\longrightarrow$ : potential regulatory link

C

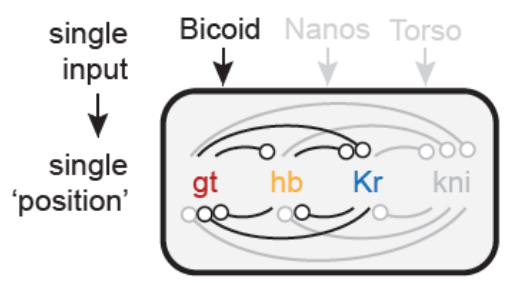

e

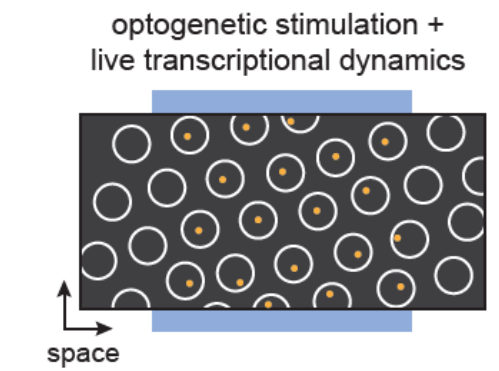

b multiple overlapping inputs

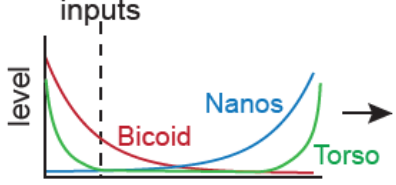

A-P position complex spatiotemporal dynamics

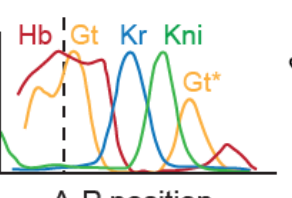

A-P position

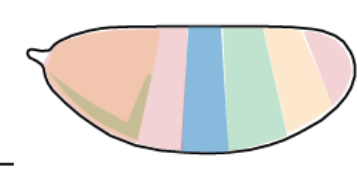

d

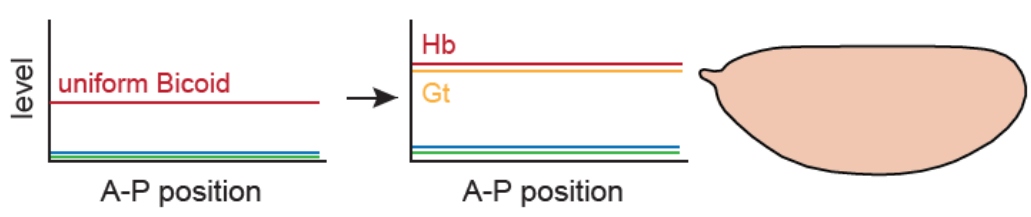

f

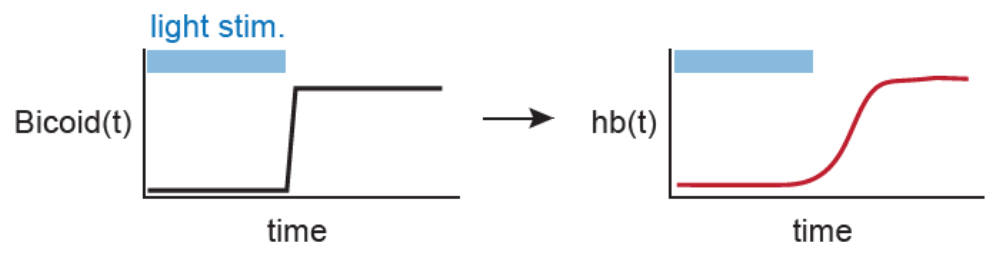

Figure 1. Studying Bicoid-dependent gap gene responses using a stimulus-response approach in single-input embryos. (a-b) The endogenous gap gene network depends on three maternally-supplied inputs (Bicoid, Nanos, and Torso) and many potential feedback and crosstalk links (in a) to generate bands of gap gene expression across the embryo (in b). A detailed understanding of this network is made challenging by the presence of multiple redundant inputs and complex dynamics as spatial patterns shift over time. (c-d) To study the effects of the Bicoid transcription factor on gap gene expression, we set out to construct a reduced-complexity network where Bicoid is the sole maternally supplied input to the network (in a) and spatial patterning is eliminated (in b), eliminating both redundancy and spatiotemporal dynamics. (e-f) To define the strength, duration, and dynamics of Bicoid-dependent gap gene responses, we acutely perturb nuclear Bicoid levels using an optogenetic technique and monitor resulting gene expression in individual nuclei using live transcription reporters. when all anterior-posterior (A-P) patterning inputs except Bicoid are eliminated, the pattern is

41 reduced and shifted relative to wildtype, but the fundamental sequence of all four gap genes is

42 maintained ${ }^{2}$. Similarly, flattening spatial patterns generates embryos that reflect a single A-P

43 position along the wild-type gradient without the complexity of network components diffusing

44 across shifting gene expression boundaries ${ }^{3,4}$. While such simplified systems can provide useful

45 insights about Bicoid-dependent features of the network, they do not distinguish between direct and indirect effects, or long- and short-term mechanisms. 
Real-time measurement of responses to acute perturbations provides a useful approach to characterize a network's dynamic features ${ }^{5,6}$. Differences in response kinetics can also distinguish direct interactions (e.g., where a transcription factor directly regulates its target's

50 expression) from indirect links (where an intermediate gene product must first be synthesized).

51 Although such rapid stimulus-response experiments have been traditionally difficult to perform

52 in vivo, the recent advent of optogenetic perturbations and live biosensors of gene expression

53 offer the possibility to dissect gene expression networks with unprecedented resolution (Figure

54 1e-f) ${ }^{6-10}$.

stimulus-response measurements in simplified embryos that lack redundant inputs and spatial patterns. We generated a series of light-sensitive Bcd variants whose nuclear-cytosolic localization can be shifted in less than a minute using blue light. We introduced these variants in embryos that lack all other sources of A-P asymmetry, eliminating the protein gradients and

61 shifting spatial distributions that typically complicate the study of patterning gene networks.

62 When constructed using optogenetic Bcd variants with different activity levels, these synthetic

63 spatially-homogeneous embryos mimic either anterior, central, or posterior embryonic positions,

64 offering a toolbox for studying the real-time transcriptional responses to acute perturbation of a

65 developmental patterning cue. Combining acute optogenetic Bcd perturbation with live-embryo

66 biosensors of gap gene expression reveals both rapid and delayed modes of Bcd-dependent

67 regulation. Anteriorly expressed gap genes $g t$ and $h b$ respond within minutes to changes in Bcd

68 concentration, consistent with a direct role for Bcd in their transcriptional activation. In contrast,

69 the medial gap gene $K r$ exhibits a delayed and inverted response, indicative of indirect Bcd- 
70 induced repression through an intermediate node. Finally, we report that the posteriorly

71 expressed gap gene $k n i$ is rapidly transcribed upon acute loss of nuclear Bcd, an unexpected

72 response suggesting that $\mathrm{Bcd}$ acts to repress kni expression without requiring new gene synthesis.

73 Our approach, combining rapid nuclear-cytosolic shuttling of a transcription factor with real-time

74 transcription measurements in a developmental gene regulatory network, offers the possibility to

75 dissect regulatory links with unprecedented precision.

\section{Results}

An activity series of optogenetic Bicoid variants with rapid stimulus-response kinetics

We engineered optogenetic variants of Bcd to serve as rapidly-switchable inputs to the gap

81 domain whose nuclear export is reversibly triggered by blue light, enabling rapid and titratable

82 optogenetic control over transcription factor activity ${ }^{11-15}$. Blue light illumination uncages a

83 buried nuclear export sequence (NES) in LEXY's C-terminal J $\alpha$ helix; in the dark, NES activity

84 is lost and Bcd's nuclear localization signal (NLS) returns the fusion protein into the nucleus

85 (Figure 2a). LEXY-based translocation typically produces a 5-fold change in nuclear protein

86 concentration ${ }^{11,13}$ whereas the natural Bcd gradient is thought to affect gene expression over a

87 larger range, suggesting that Bcd variants at various expression or activity levels may be required

88 to probe gap gene responses at different embryonic positions. We thus tested a series of Bcd-

89 LEXY variants that were either left untagged or N-terminally fused to different fluorescent

90 proteins, a modification previously observed to generate distinct Bcd activity levels (see

91 Methods $)^{16}$.

92 
bioRxiv preprint doi: https://doi.org/10.1101/2021.10.13.464280; this version posted October 14, 2021. The copyright holder for this preprint (which was not certified by peer review) is the author/funder, who has granted bioRxiv a license to display the preprint in perpetuity. It is made available under aCC-BY 4.0 International license.

a
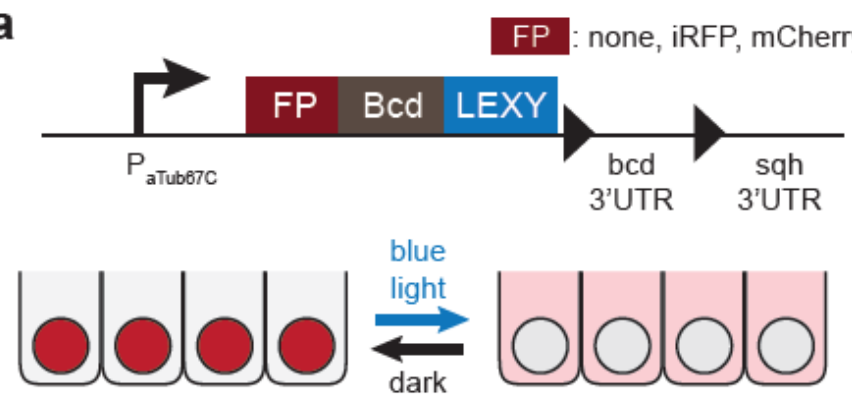

b

Bcd

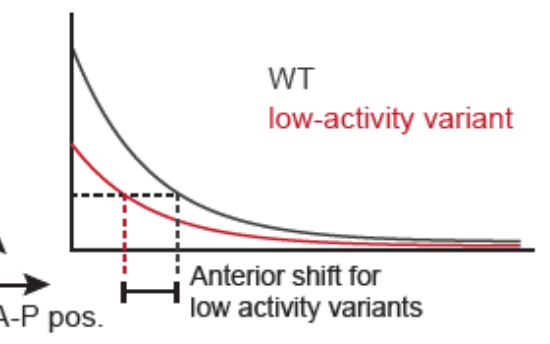

C

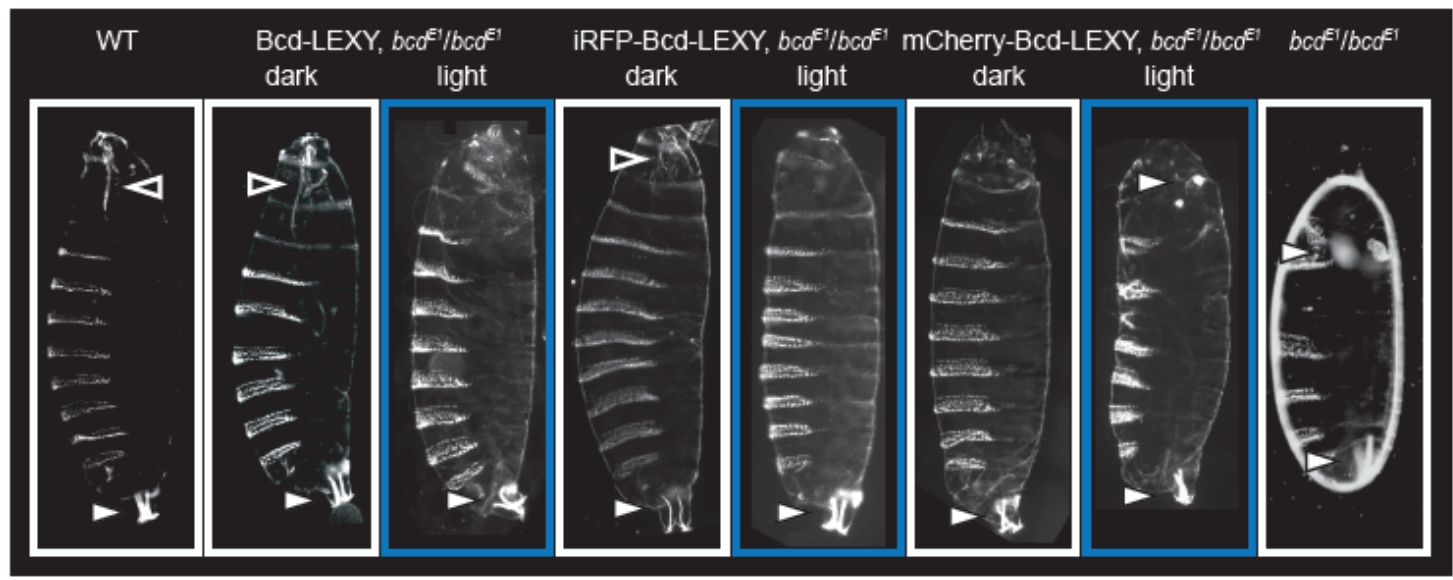

$\checkmark$ head structures $\quad$ tail structures

d

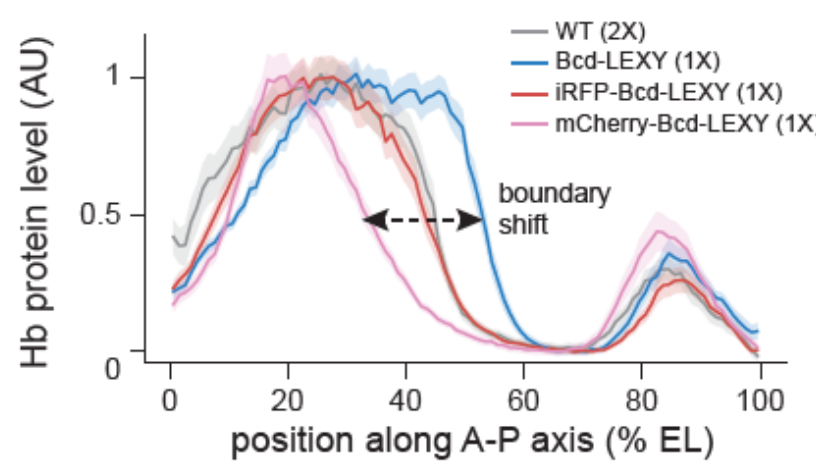

e

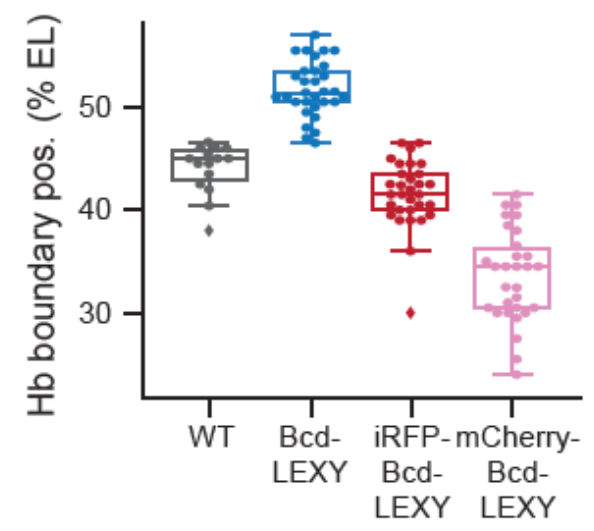

f Quantifying LEXY $\mathbf{g}$ kinetics
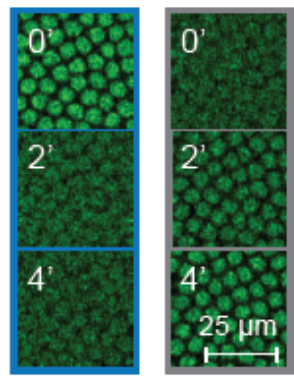

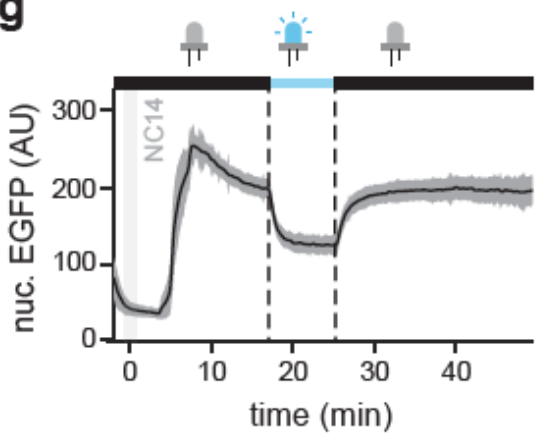

h

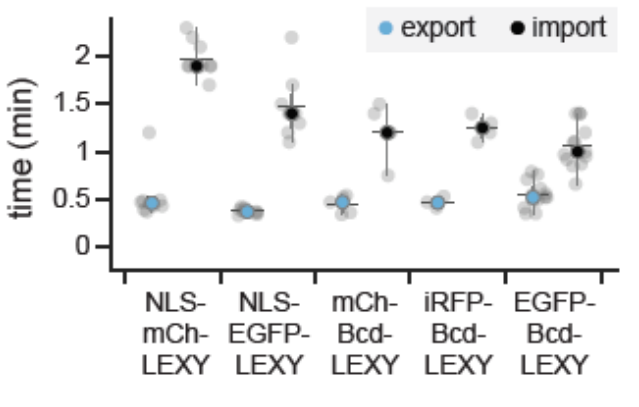


Figure 2. A series of optogenetic Bicoid variants with variable activity and rapid kinetics. (a) Bicoid was fused to various fluorescent proteins at its $\mathrm{N}$ terminus and to the LEXY optogenetic system at its $\mathrm{C}$ terminus. $450 \mathrm{~nm}$ light illumination exposes LEXY's nuclear export sequence (NES), leading to an expected decrease in Bicoid transcriptional activity. (b) Bicoid variants harboring weaker activity are expected to exhibit loss of anterior structures and an anterior shift of gene expression patterns. (c) Larval cuticles for Bcd-LEXY variants in dark and light conditions. Anterior head and posterior tail structures are indicated with the outlined and shaded arrows. Illuminated embryos exhibit loss of anterior structures or duplication of posterior structures, indicating progressive loss of Bicoid activity. (d-e) Immunofluorescence for Hunchback $(\mathrm{Hb})$ protein for three Bcd-LEXY variants, compared to WT embryos. Hb levels are quantified as a function of position and genetic background in $\mathbf{d}$, with the boundary of anterior $\mathrm{Hb}$ expression quantified for individual embryos in e. Bcd-LEXY exhibits high activity, whereas iRFP-Bcd-LEXY and mCherry-Bcd-LEXY exhibit progressively weaker activity as determined by the boundary of anterior Hb expression. (f-g) EGFP-Bcd-LEXY time course during cycle of optogenetic activation and deactivation. Representative images are shown in $\mathbf{f}$; quantification during a nuclear cycle 14 (NC14) time course of light and dark exposure shown in g. (h) Quantification of import and export kinetics for five LEXY constructs is shown in $\mathbf{h}$; similar kinetics of translocation are observed for fluorescent Bicoid variants and non-Bicoid-containing LEXY constructs.

To assess the function of each Bcd-LEXY variant, we generated embryos harboring a single variant as the sole Bcd source and assessed its function in the light and dark. Bcd is normally

97 expressed in an anterior-to-posterior gradient, so conditions in which Bicoid activity is reduced should lead to loss of anterior structures and/or an anterior shift of gap gene expression patterns

99 (Figure 2b). Bcd-LEXY and iRFP-Bcd-LEXY embryos exhibited body segmentation and cephalic furrow position consistent with high Bcd activity in the dark, and embryos harboring a

101 single copy of either allele hatched at rates of $70 \%$ and $42 \%$, respectively (Figure 2c; Table S1).

102 Blue light led to an apparent reduction in Bcd activity, characterized by the loss of mouth parts

103 and thoracic segments at the anterior and a loss of embryo viability. mCherry-Bcd-LEXY

104 carrying embryos displayed weaker overall Bcd activity, as these embryos failed to form anterior

105 structures in the dark and phenocopied $b c d^{E I}$ loss-of-function embryos under illumination

106 (Figure 2c, right panels). 
We also measured expression of the canonical Bcd target gene Hunchback $(\mathrm{Hb})$ in embryos

110 the position of the $\mathrm{Hb}$ boundary is shifted towards the posterior in Bcd-LEXY embryos and

111 progressively shifts toward the anterior in iRFP-Bcd-LEXY and mCherry-Bcd-LEXY embryos,

112 confirming that these three variants form an activity series: Bcd-LEXY > iRFP-Bcd-LEXY >

113 mCherry-Bcd-LEXY. Immunostaining for Bcd revealed that the activity differences between our

114 Bcd-LEXY variants are partially explained by differences in expression level between all three

115 variants (Figure S1a). Nevertheless, all three variants were expressed at higher levels than wild-

116 type Bcd, suggesting that fusion to LEXY and/or a fluorescent protein also partially interferes

117 with Bcd function ${ }^{16,17}$.

119 We quantitatively characterized nuclear import and export dynamics for each Bcd-LEXY

120 variant as well as two LEXY-tagged fluorescent proteins that lacked any transcription factor

121 fusions (NLS-mCherry-LEXY and NLS-EGFP-LEXY) (Figure 2f-h; see Methods for imaging

122 details). Switching $450 \mathrm{~nm}$ light on or off led to a rapid redistribution of each Bcd-LEXY variant

123 in nuclear cycle 14 (NC14) embryos (e.g., EGFP-Bcd-LEXY in Figure 2g). Comparable

124 dynamic responses were observed across all variants, with light-induced nuclear export in $30 \mathrm{sec}$

125 and darkness-induced import in 1-2 min (Figure 2h). Illumination also produced nuclear export

126 of similar magnitude and spatial precision, with a 4-fold change in nuclear concentration

127 between dark and light conditions and a spatial precision of $\sim 10-12 \mu \mathrm{m}$ (1-2 cells) (Figure S1b-

128 d). These data establish the LEXY system as a tool for rapid modulation of nuclear transcription

129 factor concentration during pre-gastrulation Drosophila embryogenesis. 


\section{A reduced-complexity embryo for dissecting Bcd regulation of gap gene expression}

Our goal is to use the fly embryo as a laboratory to measure stimulus-response functions for

133 Bicoid's regulation of gap gene expression. We thus sought to simplify the experimental system,

134 eliminating redundant inputs to the gap gene network as well as the complex spatial patterns

135 found in wild-type embryos. Recent studies ${ }^{2,3,18}$ established genetic strategies for producing

136 embryos that lack all known sources of anterior-to-posterior variation, and we used these triple-

137 mutant $b c d^{E 1} n o s^{B N} t s l^{4} / b_{c} d^{E 1} n o s^{l 7} t s l^{4}$ (henceforth referred to as $b n t$ ) embryos as a starting

138 point for introducing light-controlled Bcd-LEXY variants (see Methods for detailed information

139 on fly stock and genetics). We also reintroduced uniform levels of a weak Nanos variant (nos

140 TCEIIUC:AG) ${ }^{19,20}$ to suppress maternal $\mathrm{Hb}$ protein expression; the resulting embryos, termed

141 nos-tub bnt, are devoid of all three A-P patterning cues and produce a posterior-like gene

142 expression state throughout the embryo (Figure S2a). Onto this background we expressed a

143 single Bcd-LEXY variant at a uniform level across the embryo to serve as the sole patterning

144 input to the gap gene network ${ }^{3}$. Bcd-LEXY nos-tub bnt embryos can thus be thought of as

145 representing a single embryonic "position” set by Bcd which can subsequently be perturbed

146 using light (see e.g. Figure 3a for $K r$ in uniform mCherry-Bcd-LEXY embryos).

To define the A-P position represented by each uniformly expressed Bicoid-LEXY variant,

149 we measured the transcriptional activity for four gap genes ( $g t, h b, K r$ and $k n i)$ using the

150 MS2/MCP system (see Methods; Figure 3b-e; Figure S2b-c; Video S1-S4). We found that our

151 baseline nos-tub bnt embryos lacking all A-P patterning transcribed high levels of $k n i$ and $g t$, but

152 low levels of $K r$ and no detectable $h b$ (Figure 3b and arrow in 3f). This pattern was altered

153 dramatically in the presence of uniformly expressed Bcd-LEXY, which drove an anterior-like 
a

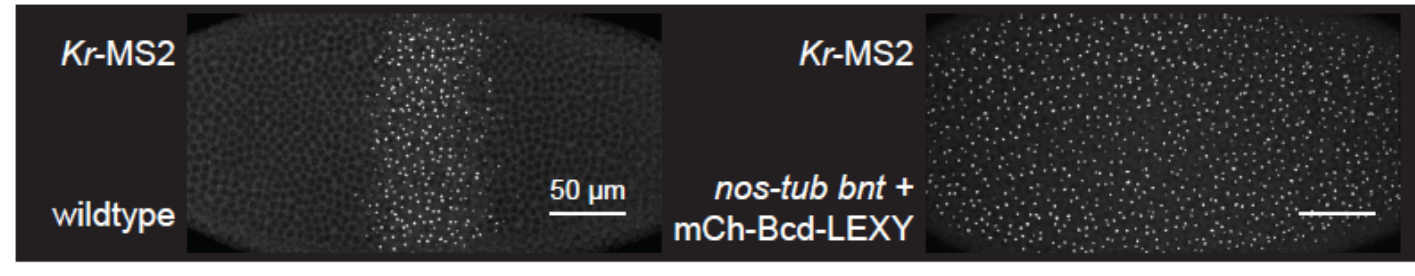

b

parental
nos-tub bnt

C

LEXY

d

+ iRFP- ${ }^{\text {dark }}$

Bcd-

LEXY

light

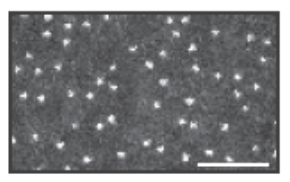

$h b$
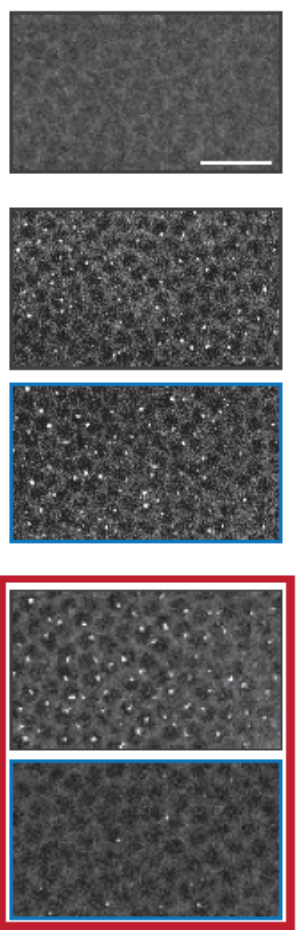

e

$+\mathrm{mCh}$ - dark

Bcd-

LEXY

light
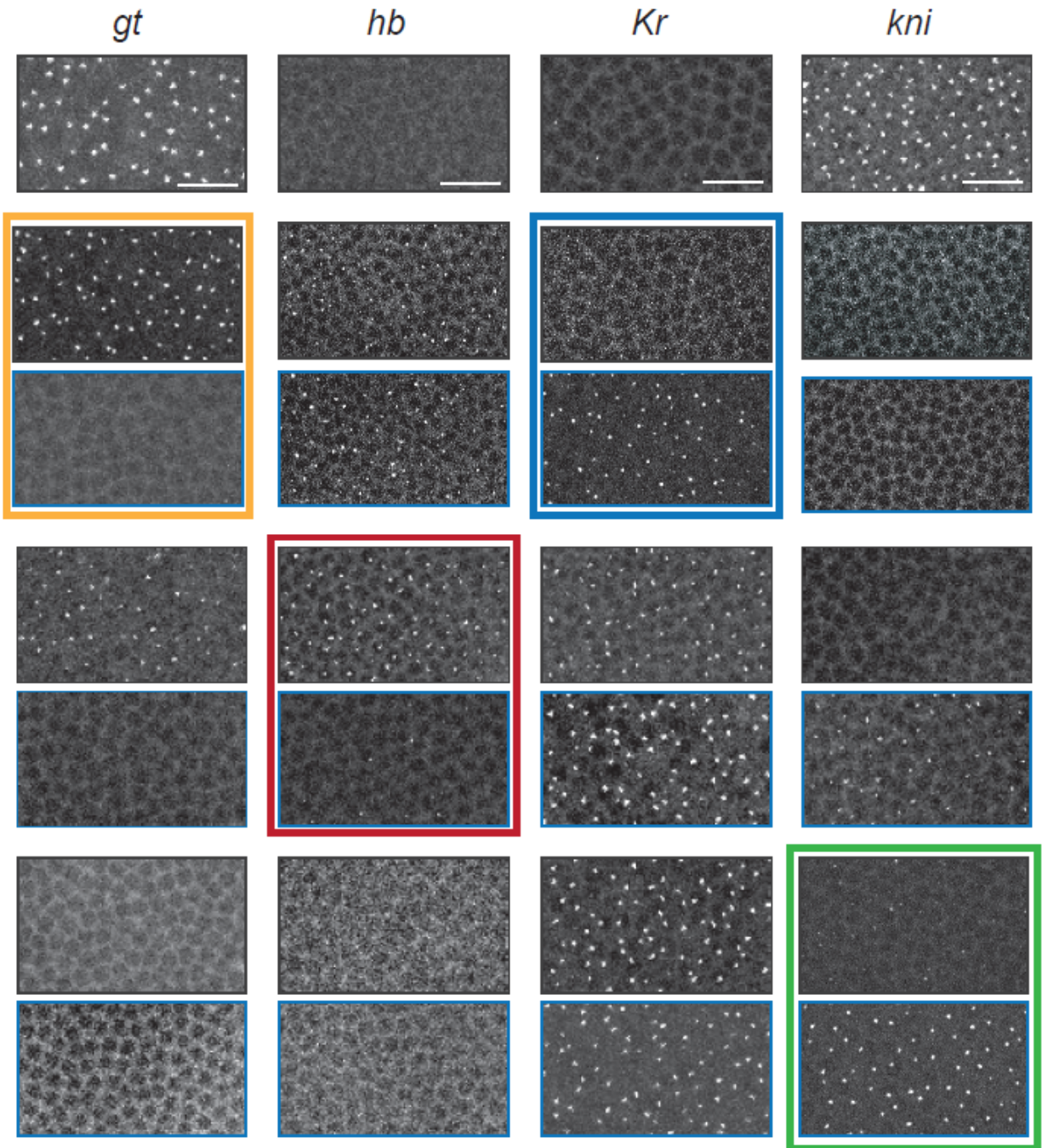

f

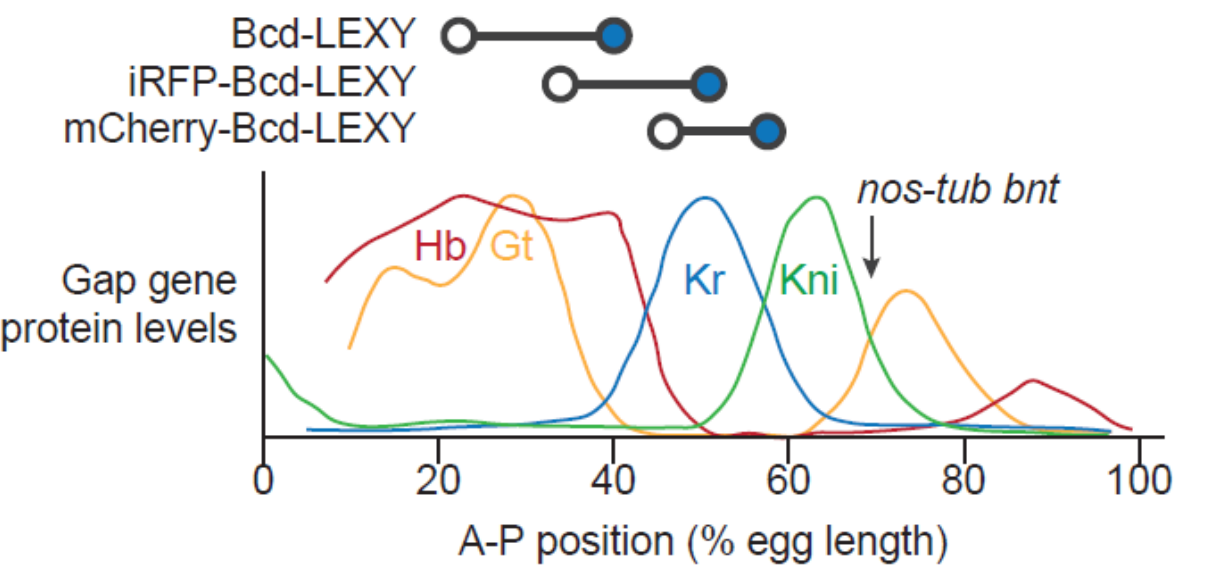


Figure 3. Spatially-uniform, single-input embryos to enable optogenetic interrogation of specific gap genes. (a) Nuclear cycle 14 embryos imaged using a $K r$ MS2 reporter. Left: embryo exhibiting wildtype A-P patterning. Right: nos-tub bnt embryo harboring a single copy of uniformly-expressed mCherryBcd-LEXY. The nos-tub bnt background eliminates all maternally supplied A-P patterns, so a uniformly expressed Bcd-LEXY variant produces a single approximate A-P position per embryo. (b-e) Regions of embryos showing MS2 reporter transcription for all four gap genes in (b) nos-tub bnt, (c) nos-tub bnt + Bcd-LEXY, (d) nos-tub bnt + iRFP-Bcd-LEXY, and (e) nos-tub bnt + mCherry-Bcd-LEXY embryos. For optogenetic illumination experiments, embryos were bathed in $450 \mathrm{~nm}$ light for $1 \mathrm{~h}$. (f) Mapping approximate embryonic positions represented by dark and light conditions in each genetic background. Bottom: diagram from Ref. 2 quantifying gap gene expression as a function of A-P position, with posterior $=100 \%$ EL. Top: the approximate position based on gap gene expression for each optogenetic variant under in illuminated (open circle) and dark conditions (blue circle).

of $g t$ and $h b$ transcription in the dark, shifting to a mid-embryo-like state of $h b$ and $K r$

transcription in the light (Figure 3c). Uniform iRFP-Bcd-LEXY embryos transcribed $g t, h b$, and

$K r$ in the dark, shifting to $K r$ and $k n i$ expression in the light (Figure 3d). Finally, mCherry-Bcd-

LEXY embryos shifted between weak and strong kni transcription depending on illumination

160 conditions, with high $K r$ transcription in both cases (Figure 3e).

3f). Illumination shifts Bcd-LEXY embryos from high $h b$ and $g t$ expression to $h b$ alone, which normally occurs between $20-40 \%$ egg length (EL); similarly, iRFP-Bcd-LEXY embryos shift from $\sim 35-50 \%$ EL, and mCherry-Bcd-LEXY embryos from $\sim 45-60 \%$ EL (Figure 3f, top).

167 Notably, parental nos-tub bnt embryos express high levels of kni and gt, consistent with a 168 position of $\sim 70 \%$ EL, because their reduced expression levels of maternal $\mathrm{Hb}$ allows that 169 expression but does not promote expression of more posterior targets in the absence of Torso/Erk 170 signaling (Figure 3f, arrow). Importantly, these results define the optogenetic Bcd variants that 171 can be used to switch each of the four core gap genes between high and low expression levels: $g t$ 172 (Bcd-LEXY), $h b$ (iRFP-Bcd-LEXY), $K r$ (Bcd-LEXY) and $k n i$ (mCherry-Bcd-LEXY) (Figure 
173 3b-e; colored boxes), which we used to interrogate the transcriptional dynamics of each gap gene

174 in the following live-imaging experiments.

\section{Anterior patterns of $\boldsymbol{h b}$ and $g t$ respond rapidly to changes in nuclear Bed concentrations}

How do gap genes respond to acute changes in nuclear Bcd concentration? To answer this question, we set out to combine optogenetic Bcd-LEXY control with live imaging of individual gap genes using the MS2/MCP system. We constructed a confocal microscope that combines a tunable 2-photon (2P) laser for GFP/mCherry imaging with a digital micromirror device and 450

181 nm LED for optogenetic stimulation (see Methods; Figure 4a; Figure S3a). 2P excitation is

182 ideal because it can be used for simultaneous GFP and mCherry imaging without triggering

183 AsLOV2 excitation (Figure S3b; Video S5-6), enabling high-resolution volumetric imaging 184 without undesirable photoactivation of our optogenetic system ${ }^{21,22}$. well as two additional constructs: an MCP-mNeonGreen protein for live transcript

188 visualization ${ }^{23-25}$ and an NLS-mCherry-LEXY indicator to define the current activity state of our 189 optogenetic system (Figure S3c-d; see Methods). By crossing females of this genotype with

190 males harboring a desired MS2-tagged gap gene reporter, we can thus deliver optogenetic stimuli

191 while imaging both LEXY nuclear translocation and transcriptional responses in individual

192 nuclei over time in live embryos (see Figure 4b using the gt-MS2 reporter).

193

194 We first performed stimulus-response measurements for Bcd regulation of anterior $h b$ 195 expression using our medium-activity iRFP-Bcd-LEXY variant (Figure 4c-d). We measured $h b$ 
bioRxiv preprint doi: https://doi.org/10.1101/2021.10.13.464280; this version posted October 14,2021. The copyright holder for this preprint (which was not certified by peer review) is the author/funder, who has granted bioRxiv a license to display the preprint in perpetuity. It is made available under aCC-BY 4.0 International license.

a

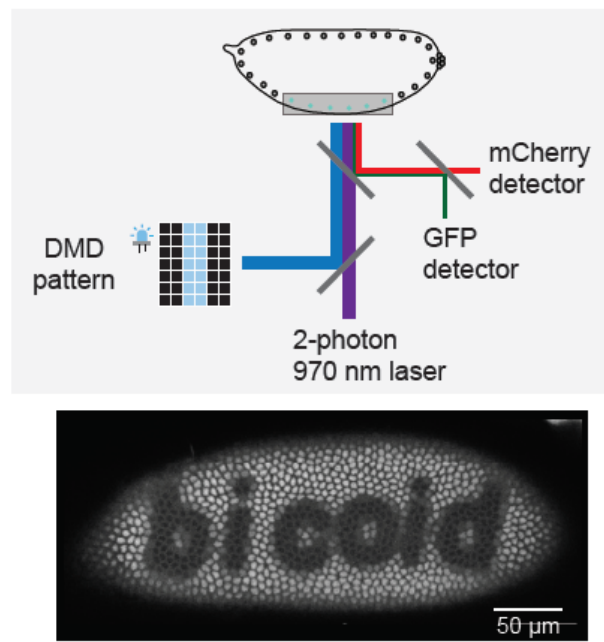

C

$h b$

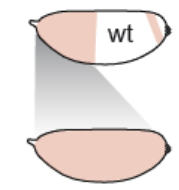

nos-tub bnt + iRFP-Bcd-LEXY

$\mathbf{f}$

gt

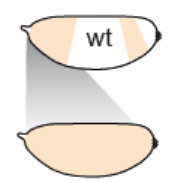

nos-tub bnt + Bcd-LEXY b
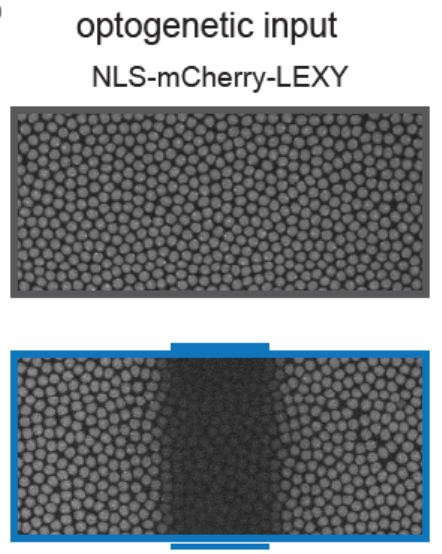

$450 \mathrm{~nm}$

illumination transcriptional response

gt MS2
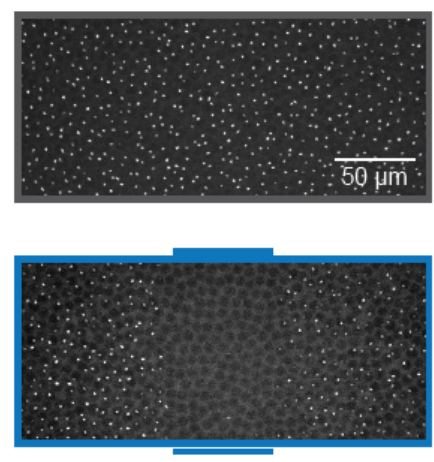

e d

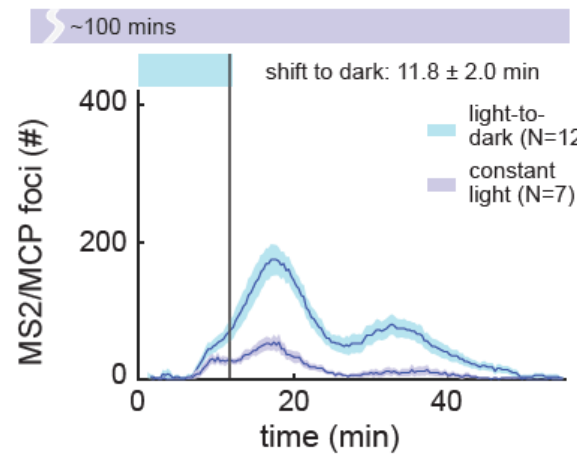

g

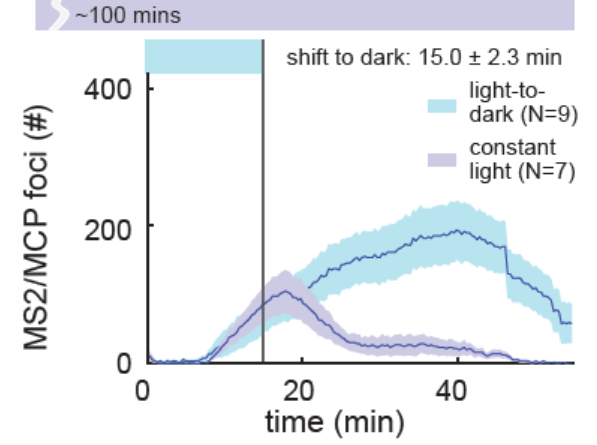

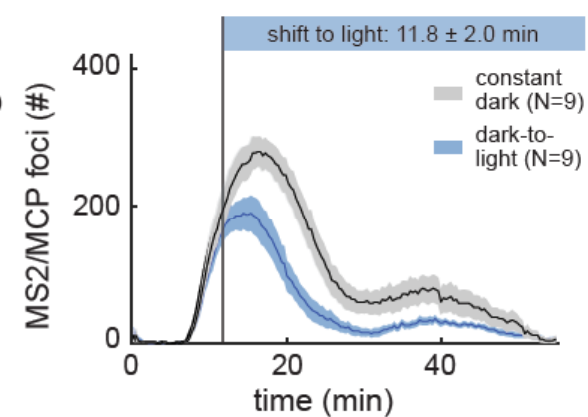

h

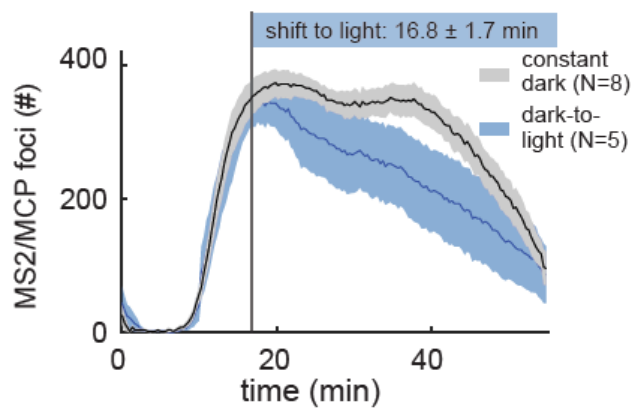

additional observations

gradual $g t$ decay from high state suggests positive autoregulation gap gene regulation

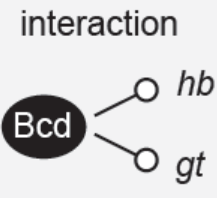

sign

1.7 - $3.3 \mathrm{~min}$

$5.9-9 \min$ 
Figure 4. Optogenetic stimulation and live transcription measurement for anterior expression patterns of $\boldsymbol{g} \boldsymbol{t}$ and $\boldsymbol{h b}$. (a) Schematic of optogenetic activation and two-photon imaging setup. $450 \mathrm{~nm}$ LED light is patterned using a digital micromirror device (DMD) to deliver optogenetic stimuli. Twophoton imaging at $970 \mathrm{~nm}$ excites GFP and mCherry without crosstalk to the LEXY optogenetic system. (b) Example of light stimulation and two-color imaging of NLS-mCherry-LEXY and MCP foci for a $g t$ MS2 transcriptional reporter. Images show ventral regions of representative embryos in the absence or presence of a $450 \mathrm{~nm}$ light input delivered in a stripe in the middle of the embryo. (c-e) Optogenetic interrogation of Bcd-induced anterior $h b$ expression dynamics. Uniformly expressed iRFP-Bcd-LEXY embryos were imaged for $h b$ MS2 reporter expression (schematic in c) upon an acute shift from light to dark (in d) and dark to light (in e); constant-light and constant-dark stimuli were sued as controls. (f-h) Optogenetic interrogation of Bcd-induced anterior $g t$ expression dynamics. Uniformly expressed BcdLEXY embryos were imaged for $g t$ MS2 reporter expression (schematic in $\mathbf{f}$ ) upon an acute shift from light to dark (in g) and dark to light (in $\mathbf{h}$ ); constant-light and constant-dark stimuli were used as controls. (i) Summary of stimulus-response results for $g t$ and $h b$. Rapid light-triggered changes in both $g t$ and $h b$ transcription are consistent with direct activation by Bcd. Subsequent gradual changes in $g t$ expression suggest the presence of positive autoregulation. For $\mathbf{d}, \mathbf{e}, \mathbf{g}$, and $\mathbf{h}$, shaded regions show standard error of the mean, and the number of embryos tested is indicated on each plot.

transcription in response to an acute increase in Bcd activity by shifting from blue light to dark conditions during early NC14; continuously illuminated embryos were used as a control (Figure

4d; Figure S4a). We found that $h b$ expression rose rapidly after a light-to-dark shift; quantifying

201 this response time revealed a shift within $1.7 \pm 0.9$ min after light perturbation (mean $\pm \mathrm{SEM}$; see

Table S2). Conversely, acute removal of Bcd by switching from dark to light conditions caused

204 data indicate that gap gene transcription can respond extremely rapidly to acute increases or as a direct transcriptional activator of anterior $h b$ expression.

How do the anterior expression dynamics of $g t$ compare to those of $h b$ ? We examined a $g t$

211 rapidly transmitted to $g t$ expression, with response times of $5.9 \pm 1.6 \mathrm{~min}$ and $9 \pm 2.7 \mathrm{~min}$ 
212 depending on the illumination sequence, indicative of direct transcriptional activation (Figure

213 4g-h; Table S2). However, unlike the case for $h b, g t$ transcription continued changing gradually,

214 only approaching a final expression state over $\sim 30 \mathrm{~min}$. A rapid initial response followed by

215 delayed progression towards a final state is suggestive of positive autoregulation ${ }^{27}$, for example

216 if $g t$ transcription were positively influenced by the past history of gap gene ( $g t$ or $h b$ )

217 expression. Indeed, positive feedback on $g t$ transcription by Gt protein was recently reported ${ }^{26}$.

218 In summary, live-embryo stimulus-response measurements identify $h b$ and $g t$ as direct Bcd

219 transcriptional targets and suggest $g t$ as a potential target of positive autoregulation (Figure 4i).

221 Optogenetic Bcd stimuli produce delayed and inverted $K \boldsymbol{r}$ transcriptional responses

222 To explore how dynamic changes in Bcd concentration alter the expression of gap genes in

223 the middle of the embryo, we next turned to the gap gene $K r$ (Figure 5a). $K r$ expression is

224 known to be regulated by multiple transcription factors, including $\mathrm{Bcd}^{28-30}, \mathrm{Stat}^{2} \mathrm{E}^{31}$, Zelda

$225(\mathrm{Zld})^{32}$, and Hunchback $(\mathrm{Hb})^{30,33}$; this complex regulation is thought to ensure that $\mathrm{Kr}$ is

226 expressed in a narrow central band, with low expression at both anterior and posterior embryonic

227 positions.

We performed stimulus-response measurements in Bcd-LEXY embryos, which exhibit stark

230 differences in $K r$ expression between constant blue light and dark conditions that reflect

231 optogenetic switching across the anterior boundary of the $K r$ pattern (Figure S4c). An acute

232 increase in nuclear Bcd-LEXY drove a corresponding decrease in $K r$ transcription, consistent

233 with our expectation of low $K r$ expression at anterior positions (Figure 5b). However, unlike $h b$

234 and $g t$, the change in $K r$ transcription only began after a $22 \pm 2$ min delay (Table S2). 
a

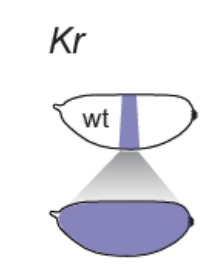

nos-tub bnt Bcd-LEXY b

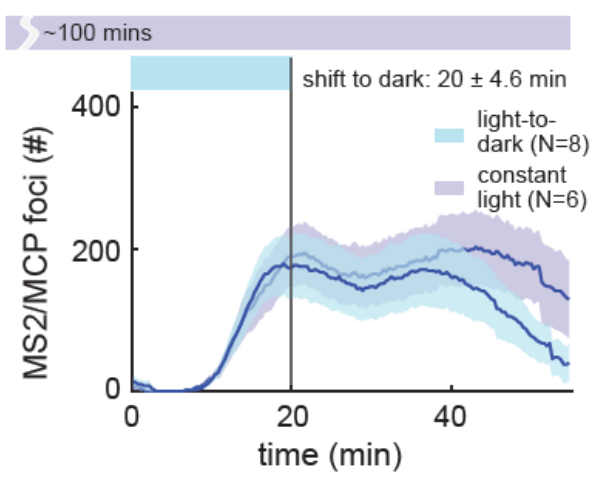

C

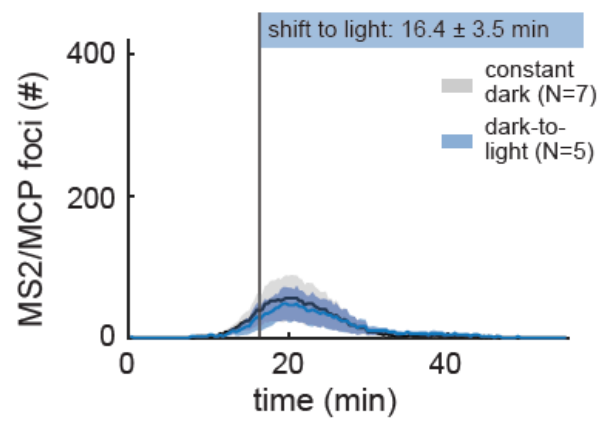

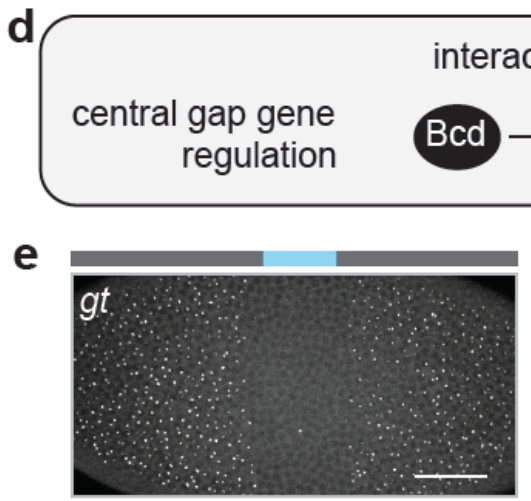

interaction sign

timescale

additional observations

delayed response suggests indirect repression, likely by $\mathrm{Gt}$

nos-tub bnt + Bcd-LEXY + MS2 gap gene reporters, imaged in NC14 under continuous illumination

Figure 5. Acute perturbation of Bcd reveals delayed negative regulation of $K \boldsymbol{r}$ transcription. (a-c) Optogenetic interrogation of Bcd-induced $K r$ transcription dynamics. Uniformly expressed Bcd-LEXY embryos were imaged using a $K r$ MS2 reporter (schematic in a) upon an acute shift from light to dark (in b) and dark to light (in c); constant-light and constant-dark stimuli were used as controls. (d) Summary of rapid perturbation results. An acute increase in nuclear Bcd-LEXY concentration drives loss of $\mathrm{Kr}$ expression after a 22 min delay. Conversely, $K r$ transcription is not observed for at least $1 \mathrm{~h}$ after an acute decrease in nuclear Bcd levels. (e) Measurement of $g t, K r$, and $h b$ transcription after continuous, local illumination in Bcd-LEXY embryos. Transcription of $g t$ is suppressed where $K r$ is transcribed, whereas $h b$ is largely unaffected in the Bcd-LEXY background. For b-c shaded regions show standard error of the mean, and the number of embryos tested is indicated on each plot.

237 Conversely, shifting the embryo from a high-Bcd state to a low-Bcd state in early NC14 did not

238 lead to any detectable change in $K r$ expression prior to gastrulation, indicating that an even

239 longer time period may be required to establish $K r$ expression upon loss of Bcd (Figure 5c). 
244 after the time needed for new protein synthesis, and repressor degradation would be required for

$245 K r$ expression to respond to a drop in Bcd activity. Our data also points to a likely candidate

246 repressor among the gap genes. We observe a tight correlation between the spatial expression

247 domains of $K r$ and $g t$ in illuminated Bcd-LEXY embryos, suggesting that Gt may act as the long-

248 lived, Bcd-induced negative regulator (Figure 5e). This model is well supported by prior

249 studies $^{34-37}$ identifying Gt as a potent repressor of $K r$ expression. In sum, our stimulus-response

250 framework can be used to measure transcription dynamics that can in turn provide insight into

251 direct and indirect links within a gene regulatory network.

252

\section{$k n i$ is transcribed rapidly upon light-triggered loss of nuclear Bed}

254 Our final target for optogenetic stimulus-response analysis was the posterior pattern of kni

256 segments, is thought to be induced by Bcd and Caudal (Cad) and repressed by $\mathrm{Hb}^{38,39}$. This

257 complex and redundant regulation involves both maternally supplied anterior inputs (e.g., Bcd-

258 dependent Cad patterning) and posterior cues (e.g., Nanos-dependent patterning of maternal Hb).

259 Interestingly, we observe high kni expression even in nos-tub bnt embryos (Figure 3b), raising

260 the question of how Bcd affects expression of a gap gene that is still highly expressed in the

261 absence of Bcd.

262

263 We examined the Bcd-dependent dynamics of $k n i$ expression ${ }^{25}$ in embryos expressing the

264 lowest activity mCherry-Bcd-LEXY variant (Figure 6b-c; Figure S4d). Acutely dropping

265 nuclear Bcd concentration led to a dramatic and unexpected change in kni transcription (Figure

266 6c). Within $2.9 \pm 0.9$ mins after a loss of nuclear Bcd, $k n i$ transcription began rising rapidly to 
a

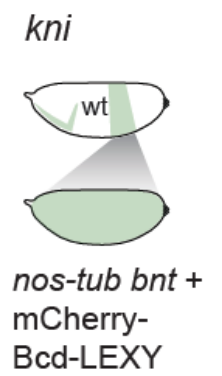

b

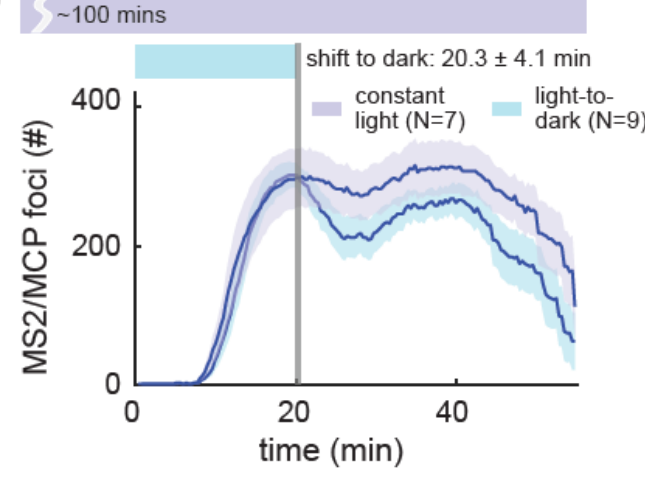

C

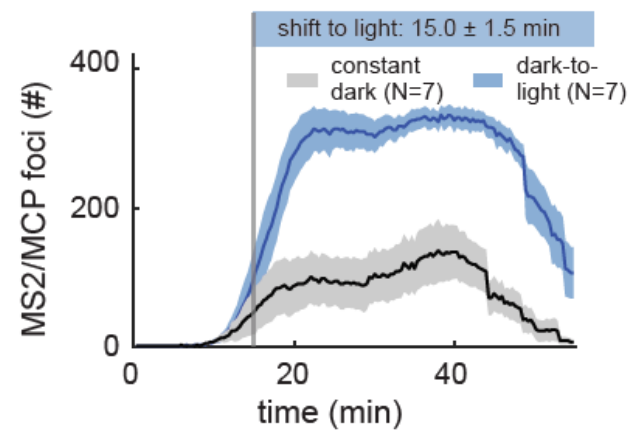

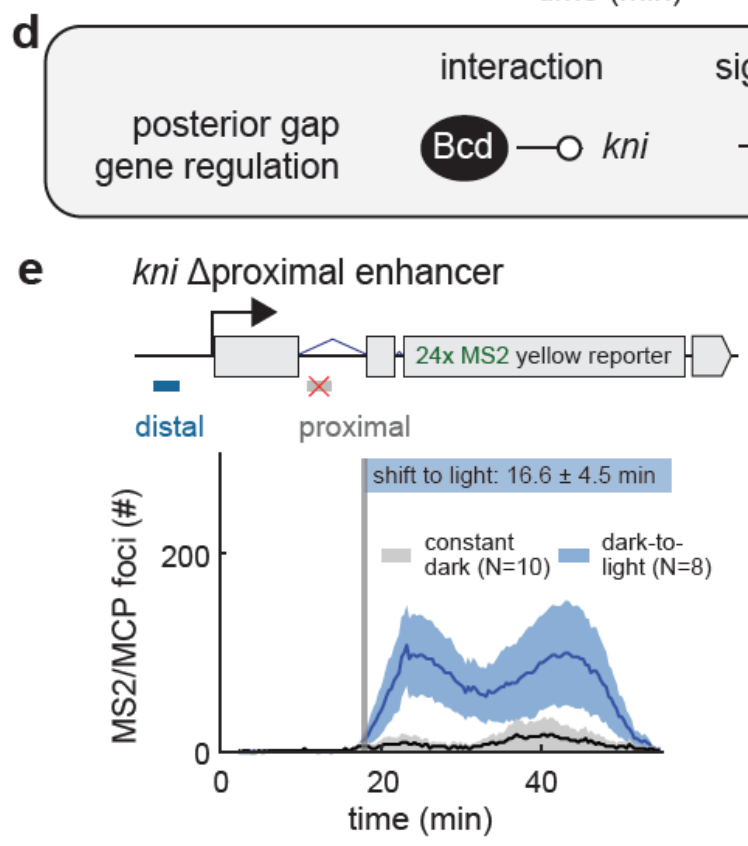

timescale additional observations

2.9 - $3.5 \mathrm{~min}$ gradual decay from high state suggests positive autoregulation

\section{f}

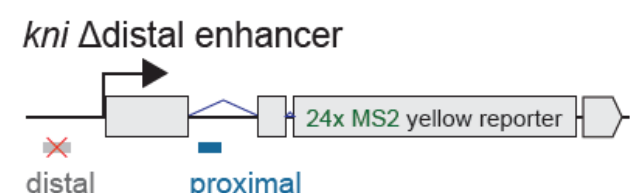

Figure 6. Acute removal of Bcd drives rapid activation of posterior $k n i$ transcription. (a-c)

Uniformly expressed mCherry-Bcd-LEXY embryos were imaged using a kni MS2 transcription reporter upon an acute shift from light to dark (in b) and dark to light (in c); constant-light and constant-dark stimuli were used as controls. (d) Summary of rapid perturbation results. An acute decrease in mCherryBcd-LEXY expression, representing a change from central to posterior Bcd levels, drives a rapid rise in $k n i$ expression. In the converse experiment, $k n i$ transcription drops rapidly but only slightly upon acute Bcd nuclear import, suggesting that $k n i$ transcription is positively autoregulated. (e-f) Experiments as in c for kni reporters in which the proximal enhancer (in $\mathbf{e}$ ) or the distal enhancer (in $\mathbf{f}$ ) were replaced with non-targeted sequence. For $\mathbf{b}-\mathbf{f}$, shaded regions show standard error of the mean, and the number of embryos tested is indicated on each plot.

269 levels that were comparable to those achieved under continuous illumination (Figure 6b).

270 Conversely, a light-induced increase in nuclear Bcd triggered a similarly rapid but smaller-

271 amplitude decrease in kni transcription (Figure 6b). Just as in the case of $g t$, the stability of the 
272 high-kni-expressing state may be indicative of positive autoregulation of kni expression by its

273 own protein product. Together, these data suggest that Bcd can act as an apparent repressor of

$274 k n i$ expression, an unexpected role for Bcd which is typically considered to perform only

275 transcriptional activation functions. The initiation of $k n i$ transcription within 2 min after Bcd

276 nuclear export is only compatible with a direct regulatory link, not Bcd-induced expression of an

277 intermediate repressor.

To gain further insight into the repressive effect, we set out to define its requirements in the

$280 k n i$ enhancer regions. The posterior pattern of kni expression is known to be regulated by two

281 enhancers, an 818 bp proximal enhancer and a $2.3 \mathrm{~kb}$ distal enhancer ${ }^{38,40}$. We generated embryos

282 expressing kni MS2 reporters with either the proximal or distal enhancer sequence replaced with

283 non-regulated sequence ${ }^{41}$ and monitored expression in response to acute Bcd removal (Figure

284 6e-f). We found that the $k n i$ reporter lacking the proximal enhancer ( $k n i \Delta$ proximal reporter) still

285 showed potent regulation by mCherry-Bcd-LEXY, whereas the kni $\Delta$ distal reporter was not

286 affected by light-induced changes in nuclear Bcd (Figure S4e-f; Video S7-8). Transcription

287 from the kni $\Delta$ proximal reporter also rose rapidly upon the shift to blue light, matching what was

288 observed from the wild-type regulatory sequence (Figure 6c,e). Our results are consistent with

289 prior observations that the kni-distal enhancer exhibits higher Bcd binding than does the kni-

290 posterior enhancer, arguing that Bcd exerts its regulatory effects at the distal enhancer ${ }^{38,40}$. In

291 summary, our acute stimulus-response framework identifies a rapid, repressive role for Bcd in

292 regulating kni transcription through the kni distal enhancer, underscoring the power of

293 optogenetic perturbation in a simplified genetic context to identify both known and unknown

294 gene regulatory relationships. 


\section{Discussion}

296 A stimulus-response strategy for dissecting complex developmental gene networks

297 We have described a combined genetic and optogenetic strategy to gain insight into one

298 canonical developmental patterning network: control of gap gene expression by the Bicoid 299 morphogen during Drosophila embryogenesis. Our strategy relies on three advances. First, we

300 experimentally simplify the conditions under which the gap gene network operates, eliminating

301 all pre-existing landmarks along the anterior-posterior axis to produce embryos with uniform

302 positional identity. Although the reduced network involves just one input transcription factor

303 (Bicoid) and four output genes (anterior giant, anterior hunchback, central Krüppel, and posterior

304 knirps), it captures much of the complexity of the wildtype pattern including stripes of gap gene

305 expression when Bcd is delivered in a head-to-tail gradient ${ }^{2}$. Second, we re-introduce

306 optogenetic Bcd variants to shift these uniform embryos to any of three distinct anterior-posterior

307 positions, enabling us to experimentally isolate specific gap genes patterns. Finally, we combine

308 acute optogenetic perturbation with live-cell biosensors of target gene expression to map the

309 network's responses to acute changes in transcription factor concentration over time. Doing so

310 required establishing new imaging methods for two-color confocal imaging and optogenetic

311 activation in vivo, a challenge we solved by combining $970 \mathrm{~nm}$ two-photon imaging of

$312 \mathrm{GFP} / \mathrm{mCherry}$ with $450 \mathrm{~nm}$ excitation of the LEXY optogenetic system.

\section{Bcd-dependent regulation of anterior and posterior gap gene patterns}

315 Our optogenetic stimulus-response experiments broadly support the canonical view of Bicoid 316 as a direct transcriptional activator of $g t, h b$, and $K r$. We find that both $g t$ and $h b$ are transcribed

317 rapidly upon acute Bicoid nuclear import (Figure 4) and $K r$ expression requires the presence of 20 
318 low Bicoid activity (Figure 5). Our data also point to multiple regulatory links between gap

319 genes. We find that $g t$ expression is sustained when nuclear Bicoid is acutely removed (Figure

320 4), consistent with reports of its positive autoregulation ${ }^{42}$; that $K r$ exhibits delayed negative

321 regulation by Bicoid (Figure 5), likely through Gt as an intermediate node ${ }^{43}$; and that kni

322 expression remains high even after Bicoid nuclear import (Figure 6), consistent with its positive

323 autoregulation ${ }^{44}$. Importantly, each of these network connections can be identified using a single,

324 unified experimental workflow: acute optogenetic Bicoid perturbation and live recording and

325 quantification of a target gene's transcriptional dynamics.

Our study also revealed an unexpected result: rapid initiation of kni transcription after acute

328 removal of mCherry-Bicoid-LEXY from the nucleus. Bicoid is not expected to act as a

329 transcriptional repressor, so it is surprising to find any context in which its removal triggers rapid

330 initiation of transcription. Classical models interpret loss of $k n i$ at anterior positions as being the

331 consequence of indirect Bicoid-dependent regulation: repression by anterior gap gene products

332 (e.g. $h b$ or $K r$ ) or weak activation by Caudal, which is translationally repressed by Bicoid ${ }^{38,45,46}$.

333 Our result appears inconsistent with all of these explanations, as kni expression rises near-

334 instantaneously after mCherry-Bicoid-LEXY nuclear export (Figure 6), too rapidly for changes

335 in gap gene or Caudal protein levels to occur. Furthermore, rapid kni de-repression requires the

336 distal enhancer, the predominant site of Bcd binding ${ }^{38,40}$.

338 How might rapid transcriptional activation occur upon loss of nuclear Bicoid? Our data is

339 consistent with many possible mechanisms. Bicoid may compete for binding to the kni distal

340 enhancer with another more potent transcriptional activator, such that Bicoid loss paradoxically 
341 increases kni expression. Alternatively, Bicoid may cooperatively associate with a transcriptional

342 repressor at the kni enhancer and lead to increased repressor binding, mirroring the well-

343 established interaction between Dorsal and Groucho for repressing subsets of genes along the

344 dorsoventral axis ${ }^{47,48}$. We look forward to future studies that precisely define Bcd's repressive

345 role in kni expression, as well as extension of our acute stimulus-response methods to other

346 complex gene regulatory networks.

348 Optogenetic stimuli have recently found widespread use in developmental contexts, from

349 identifying critical time windows for developmental decisions ${ }^{13,49-52}$ to erasing and replacing

350 signaling gradients with spatial light patterns ${ }^{53}$. However, optogenetic control on its own is not a

351 panacea for revealing the inner workings of complex biological networks. Here we show that

352 optogenetics can be used at a more granular level to home in on dynamic relationships between a

353 transcription factor and its target genes in vivo. Nevertheless, work on an experimentally reduced

354 system only constitutes a first step in understanding the full gap gene network, and we look

355 forward to future studies that examine Bicoid-dependent responses as other factors from the

356 natural system are systematically re-introduced. We can also envision extending the current

357 approach to perturbing multiple nodes (e.g., by constructing LEXY fusions of all gap genes);

358 quantitative modeling ${ }^{4}$ could elaborate network architecture still further. The future is bright for

359 optogenetic interrogation of developmental gene networks. 


\section{$361 \quad$ Material and methods}

362 Plasmids. Constructs were generated using In-Fusion assembly (Clontech) and oligonucleotides

363 for primers were obtained from Integrated DNA Technologies. Constructs are available via

364 Addgene or on request. Bcd-LEXY constructs are generated from pCol- $\alpha$ Tub67C-EGFP-Bcd-

365 FRT-bcd 3'UTR-3xP3-RFP-FRT-sqh 3'UTR ${ }^{54}$, where the N-terminal EGFP was either removed

366 or replaced by iRFP or mCherry and LEXY domain was inserted as C-terminus with a 15 amino-

367 acid long linker in between. NLS-mCherry-LEXY constructs are generated by ligation of the

368 NLS-mCherry-LEXY insert part PCR amplified from a mammalian expression vector (Addgene

$369 \# 72655^{11}$ ) and a fly expression vector pBabr-mTub-MCS-sqh3'UTR (courtesy from Wieschaus

370 lab) digested by restriction enzymes NheI and SpeI. mCherry was subsequently replaced by

371 EGFP to generate NLS-EGFP-LEXY plasmid.

372

373 Fly stocks and genetics. Establishing Bcd-LEXY and $b c d$ nos tsl fly stocks: For generation of

374 transgenic flies and stocks, all four Bcd-LEXY (Bcd-LEXY, EGFP-Bcd-LEXY, iRFP-Bcd-

375 LEXY and mCherry-Bcd-LEXY) constructs were integrated into the third chromosome using the

$376 \phi C 31$-based integration system ${ }^{55}$ at the VK33 site (65B2) by BestGene. NLS-mCherry-LEXY

377 and NLS-EGFP-LEXY constructs were integrated into the second chromosome at the VK02 site

378 (47C6). Each Bcd-LEXY variant was then further recombined either with $b c d^{E 1}$ or $b c d^{E 1} n o s^{B N}$

$379 t s l^{4}$ on the third chromosome ${ }^{54}$. NLS-mCherry-LEXY was recombined with MCP-mNeonGreen

380 on the second chromosome, and further crossed with $b c d^{E l}{ }_{n o s}{ }^{l 7} t s l^{4}$ on third chromosome to

381 generate MCP-mNeonGreen NLS-mCherry / Cyo; bnt / TM3 flies. 
We obtained few and poor-quality embryos from $b c d^{E 1} n o s^{B N} t s l^{4}$ homozygous females, and thus used $b c d^{E 1} n o s^{B N} t s l^{4} / b c d^{E 1} n o s^{l 7} t s l^{4}$ transheterozygotes for further experiments. While $n o s^{B N}$ is a complete loss of both nos RNA and protein, which impedes both pole cell migration

386 and therefore germline cells formation and abdominal segmentation, $\operatorname{nos}^{l 7}$ is a partial deletion

387 near C-terminal of the zinc-finger domain that maintains normal germline development.

388 However, in terms of body segmentation phenotype and gap gene expression pattern, nos ${ }^{B N}$ and

$389 \operatorname{nos}^{l 7}$ both exhibit indistinguishable patterns as expected from severe loss of function ${ }^{56,57}$,

390 supporting the use of this transheterozygous background for nanos loss of function in A-P

391 patterning.

393 Establishing uniform Bcd-LEXY embryos: To achieve uniform Bcd expression, Bcd-LEXY, bnt

394 flies were crossed to heat shock-inducible flippase expressing flies and the resulting larvae were

395 heat shocked at $37^{\circ} \mathrm{C}$ for three continuous days for 1 hour each day. After one generation of

396 outcrossing, progeny lacking the bcd 3'UTR were sorted by loss of RFP expression. Then Bcd-

397 LEXY variants were driven by sqh 3'UTR (see Figure 2A) resulting in a uniform distribution of

398 Bcd along the AP axis.

400 Establishing nos-tub bnt uniform Bcd-LEXY embryos: In wild-type embryos, nanos mRNA is

401 localized at the posterior pole and produces a posterior-to-anterior gradient of Nanos protein ${ }^{19}$. A

402 second population of nanos mRNA is not asymmetrically patterned and produces uniform Nanos

403 protein that plays a crucial role in suppressing maternal Hunchback translation ${ }^{20}$. Complete loss

404 of nanos disrupts both the patterned and uniform contributions, leading to abnormally high levels

405 of Hunchback throughout the embryo. We thus used a nos-tub:TCEIIUC:AG construct ${ }^{20}$ 
406 (courtesy of the Gavis lab) as a uniformly-expressed, reduced-activity form of Nanos to reduce

407 maternal $\mathrm{Hb}$ levels, thereby allowing expression of abdominal gap genes like $k n i$ and $g t$. The

408 construct was further recombined to the Sp marker on the same chromosome to mark the

409 transgene, and then crossed with male uniform Bcd-LEXY $b c d^{E 1} n o s^{B N} t s l^{4} /$ TM3 flies to

410 generate nos-tub:TCEIIUC:AG /+; uBcd-LEXY $b c d^{E 1} n o s^{B N} t s l^{4} /$ TM3 flies. By crossing males

411 of the preceding genotype to MCP-mNeonGreen, NLS-mCherry / Cyo; $b c d^{E 1} \operatorname{nos}^{l 7} t_{s l^{4}} / \mathrm{TM} 3$

412 females, we obtained female flies with the genotype nos-tub:TCEIIUC:AG / MCP-mNeonGreen

413 NLS-mCherry; uBcd-LEXY $b c d^{E 1} n o s^{B N} t s l^{4} / b c d^{E 1} n o s^{l 7} t s l^{4}$ that we then caged with MS2

414 reporter males for live imaging.

416 Cuticle preparation. For dark and light conditions, embryos with specific Bcd-LEXY variants

417 were collected between $0-1 \mathrm{~h}$ post laying in the dark on an agar plate. Then embryos for the light

418 condition were placed under a custom-built panel of blue LEDs and removed from light after

419 4hrs. In dark conditions, embryos were kept in a light-tight box away from ambient room light or

420 blue light to prevent inadvertent optogenetic stimulation. After a $3 \mathrm{~h}$ incubation in light or dark

421 conditions, embryos were kept at room temperature (at $22^{\circ} \mathrm{C}$ ) for another $24-36 \mathrm{~h}$ and then

422 bleached, then moved to the methanol-heptane glass tube and vigorously shaken for $20 \mathrm{sec}$.

423 Embryos settled at the bottom were removed and placed on a glass slide with Hoyer's solution

424 (1:1 premix lactic acid) and sandwiched between the glass slide and cover glass. The slide was

425 placed at $65^{\circ} \mathrm{C}$ overnight and then imaged on a Nikon Eclipse Ni dark-field microscope at 10x

426 zoom. 
428 Immunostaining and imaging. Embryos were collected every $2 \mathrm{~h}$ and aged in dark for another

4292 h. Embryos were dechorionated by bleaching and then heat fixed and stained essentially as

430 described in ${ }^{1,54}$ with rabbit anti-Bcd, mouse anti-Hb primary antibodies (courtesy by Eric

431 Wieschaus) and sheep anti-GFP (Invitrogen, USA) followed by fluorophore-conjugated

432 secondary antibodies Alexa-488 (sheep), Alexa-555 (mouse), and Alexa-647 (rabbit) from

433 Invitrogen. For pairwise comparisons of wild-type and mutant backgrounds, embryos expressing

434 HisGFP collected the same way were mixed in each tube for staining and imaging. Stained

435 embryos were imaged on a Nikon A1R laser-scanning confocal microscope, and a $5 \mu \mathrm{m}$ z-stack

436 around the midsagittal plane with step size of $1 \mu \mathrm{m}$ were taken. For data analysis, custom

437 MATLAB imaging analysis code recognized the contour of the embryo and extracted the

438 intensity of the surface nuclei for all three channels. Intensities of three channels were

439 normalized to HisGFP embryos that mixed in each slide respectively, with 1 being the mean

440 maximal intensity of HisGFP embryos. For Hb level, min-max normalization was further

441 conducted for clear comparison of boundary position, and half maximal positions of the posterior

442 boundary of anterior expression domain were picked out for each genotype for the box plot.

444 Two-photon microscopy. A custom microscope was built to simultaneously perform two-

445 photon excitation imaging and localized optogenetic stimulation on the same setup. A

446 Chameleon Ultra II tunable laser was used at $970 \mathrm{~nm}$ to simultaneously excite green and red-

447 tagged biomolecules ${ }^{17,58,59}$. The laser beam was collimated and passed through a laser power

448 modulator Pockels cell (350-80-LA-02 KD P Series E-O Modulator, Conoptics, USA). The

449 output laser beam was expanded to $4 \mathrm{~mm}$ diameter (AC254-050-AB-ML, AC254-150-AB-ML,

450 Thorlabs, USA) before reaching a two-axis scan galvo mirror $(6210 \mathrm{H}$, Cambridge Technology, 
451 USA). After the scan mirrors, the laser beam passed through a f-theta lens (focal length $63 \mathrm{~mm}$;

452 4401-388-000-20, Linos, USA), a tube lens (focal length $180 \mathrm{~mm}$, AC508-180-AB-ML,

453 Thorlabs) and focused on the imaging sample using a high numerical aperture objective (NA,

454 Nikon 1.3 NA, 40X). The fluorescence signal was collected and sent to two sensitive point photo

455 multiplier tubes (H10770A-40, Hamamatsu, Japan). The microscope setup interfaces via data

456 acquisition cards (DAQ; PCIe 6321 and PCIe 6374, National Instruments, USA) using

457 MATLAB-based ScanImage 5.6 software $^{60}$. For live imaging, embryos were imaged close to

458 cover glass surface (image resolution $1024 \times 512$ pixels at $3.2 \mu$ s pixel dwell-time; see imaging

459 details in Table S3).

460

461 Optogenetic stimulation. LEXY perturbation was achieved using a digital micro-mirror device

462 (DMD; DLP 4500 LightCrafter, Texas Instruments, USA) to project spatial patterns and to

463 rapidly change light levels ${ }^{5}$ (see Figure S2a) through a parallel light path using a long-pass 473

464 nm dichroic mirror and a combination of color and interference filters to attenuate the DMD's

465 blue LED wavelength (445 $\pm 8 \mathrm{~nm}$ ). To synchronize two-photon image acquisition and DMD

466 blue light activation cycles, an external trigger mode in DLP LightCrafter control software was

467 used. The software controls the LED light wavelength, pulse duration, pulse duty cycle, the

468 number of pulses, and the type of spatial image pattern to project on the imaging sample (see

469 details in Table S3). The optimum blue light level for optogenetic perturbation was determined

470 by optimizing the maximum protein export with minimal light scattering to neighboring nuclei

471 (see Figure 2e). After scanning the range between $50-250 \mu \mathrm{W} / \mathrm{cm}^{2}$ of blue-light on/off pulsatile

472 cycles of LEXY-tagged protein nuclear signal (data not shown), $100 \mu \mathrm{W} / \mathrm{cm}^{2}$ was determined for 473 all optogenetic perturbations performed in this study. 
475 Live imaging data collection. For the live data acquisition and light perturbation experiments,

476 flies were kept in dark at $25^{\circ} \mathrm{C}$ and the embryos were collected on an agar plate between $1-2 \mathrm{hrs}$

477 post laying. For live imaging, embryos were dechorionated on double-sided tape and mounted on

478 a glued membrane film (Lumox film, Starstedt, Germany). Followed by covering them in

479 halocarbon oil 27 and sandwiched between the membrane and the cover glass slide (cover glass

480 washed and cleaned with pure ethanol, slide \#1.5, Sigma BR470045). The data collection was

481 performed using a custom-built two-photon microscope using $970 \mathrm{~nm}$ laser excitation for green

482 (EGFP and mNeonGreen tagged protein and MS2 loops respectively) and red (only the NLS-

483 mCherry-LEXY) at room temperature (ranges from $21.5-22.5^{\circ} \mathrm{C}$ ). And the blue light

484 perturbation was done by a DMD unit installed on the same setup; see (see microscope section).

485 Summarized details on data collection for specific experiments are tabulated in Table S3.

Data quantification and statistical analysis

488 Bed-LEXY activity. To estimate the functional Bicoid activity (potency) of fluorescently tagged

489 Bcd-LEXY fusion proteins (as well as remaining activity in $b c d^{E l}$ homozygous mutant fly lines

490 and the Bcd dose level), we used protein immunostaining of the Bcd target gene $\mathrm{Hb}$ and

491 measured position shifts in the posterior Hb boundary as well as position shifts of the cephalic

492 furrow. All shifts were scaled according to embryo length and the quantified estimates are

493 presented in Figure 2d-e (Table S1 for cephalic furrow position shifts).

494

495 LEXY tagged protein export and import kinetics. Blue light-induced LEXY export kinetics

496 and nuclear localization signal (NLS)-induced import kinetics were determined by analyzing the 
nuclear intensity of the fluorescent moiety of these fusion proteins. Intensity time traces were

498 averaged and fitted with a single exponential $n 0 * \exp (-t / \tau)$ to estimate the export rate, i.e. the

499 inverse of the time constant. Similarly, the import time constants were estimated using n $0 *(1-$

$500 \exp (-\mathrm{t} / \tau))$ as the fitting model (Figure 2f-h). Note: the uniform EGFP-Bcd-LEXY, NLS-

501 mCherry-LEXY, and NLS-EGFP-LEXY lines were measured using the DMD-equipped custom-

502 built two-photon microscope, while the uniform mCherry-Bcd-LEXY and iRFP-Bcd-LEXY

503 lines were imaged on a commercial Nikon A1R confocal microscope (imaging conditions can be

504 found in Table S3). For LEXY translocation kinetics fits, the mean and the standard deviation

505 are presented for multiple embryo replicates.

507 Quantification of the reporter gene MS2 spots. We created a custom MATLAB script to

508 analyze and visualize time-lapse MS2 counts. In brief, two-color raw tiff image data was

509 acquired on a custom-built two-photon microscope (MS2 data acquisition setting details can be

510 found Table S3; see Figure S3 and Figure S4). The red channel NLS-mCherry-LEXY nuclear

511 signal was segmented, and intensity traces were used to estimate nuclear cycle start and the

512 optogenetic perturbation state during MS2 imaging. The rise in NLS-mCherry-LEXY signal time

513 was set to $5.6 \mathrm{~min}$ in $\mathrm{NC} 14$ (this $\mathrm{x}$-axis time offset was set for C14 start for both red and green

514 channels; see Figure S3d). For MS2 data, the image data was z-max projected (8 total z slices,

515 each $1.1 \mu \mathrm{m}$ apart), then a 2D gaussian filter was applied to filter-out small structures and

516 followed by threshold to select MS2 spots. Finally, every spot was counted as an active MS2

517 spot in the center part of the embryo $\left(40 \mathrm{x} 150 \mu \mathrm{m}^{2} \mathrm{ROI}\right)$ for either blue light illuminated or dark

518 conditions embryos. All the data representing spot count time traces indicate the mean and

519 standard error of the mean over multiple embryo measurements, unless stated otherwise. 
521 Mean response time post light perturbation. For response time quantification, we measured

522 the difference between individual MS2 foci count time traces (On-Off) with mean MS2 foci

523 count time traces (On) light condition (Figure 4b light blue color individual traces subtracted by

524 mean purple trace). Followed by taking gradient (25\% amplitude change) and embryos showing

525 no change or below the threshold $(<25 \%)$ were excluded from the analysis. Finally, the response

526 mean-time and standard error of the mean were reported in Table S2 (taking account of error in

527 light on/off time by using error propagation).

\section{Acknowledgements}

530 The authors thank members of the Gregor and Toettcher laboratories, Liz Gavis, Mustafa

531 Khammash, Sant Kumar, Jason Puchala, and Trudi Schüpbach. The project was supported by

532 NSF grant PHY-1734030 (T.G.) and CAREER-1750663 (J.E.T.); NIH grants R01GM097275

533 (T.G.), U01DA047730 (T.G.), U01DK127429 (T.G. and J.E.T.), T32GM007388 (M.S.); and a

534 Princeton MOL Innovation Award (J.E.T.). We also acknowledge imaging support from the

535 Princeton Molecular Biology Microscopy Facility, which is a Nikon Center for Excellence.

\section{References}

5381 Briscoe, J. \& Small, S. Morphogen rules: design principles of gradient-mediated embryo patterning. Development 142, 3996-4009, doi:10.1242/dev.129452 (2015).

5402 Petkova, M. D., Tkacik, G., Bialek, W., Wieschaus, E. F. \& Gregor, T. Optimal doi:10.1016/j.cell.2019.01.007 (2019). states induced by the bicoid morphogen gradient. Elife 6, doi:10.7554/eLife. 28275 (2017). 
5464 Jaeger, J. et al. Dynamic control of positional information in the early Drosophila

$547 \quad$ embryo. Nature 430, 368-371, doi:10.1038/nature02678 (2004).

5485 Wilson, M. Z., Ravindran, P. T., Lim, W. A. \& Toettcher, J. E. Tracing Information Flow from Erk to Target Gene Induction Reveals Mechanisms of Dynamic and Combinatorial Control. Mol Cell 67, 757-769 e755, doi:10.1016/j.molcel.2017.07.016 (2017).

6 Rullan, M., Benzinger, D., Schmidt, G. W., Milias-Argeitis, A. \& Khammash, M. An Optogenetic Platform for Real-Time, Single-Cell Interrogation of Stochastic Transcriptional Regulation. Mol Cell 70, 745-756 e746, doi:10.1016/j.molcel.2018.04.012 (2018).

$7 \quad$ Lorena de Mena, Patrick Rizk \& Rincon-Limas, D. E. (Front Genet., 2018). Patel, A. L. et al. Optimizing photoswitchable MEK. Proc Natl Acad Sci U S A 116, 25756-25763, doi:10.1073/pnas.1912320116 (2019). nuclear protein export. Nat Commun 7, 10624, doi:10.1038/ncomms10624 (2016). inductive cues on the way to gastrulation. Proceedings of the National Academy of Sciences of the United States of America 118, doi:10.1073/pnas.2102691118 (2021). Farahani, P. E., Reed, E. H., Underhill, E. J., Aoki, K. \& Toettcher, J. E. Signaling, Deconstructed: Using Optogenetics to Dissect and Direct Information Flow in Biological Systems. Annual Review of Biomedical Engineering, doi:10.1146/annurev-bioeng083120-111648 (2021). SY, C. et al. Optogenetic Control Reveals Differential Promoter Interpretation of Transcription Factor Nuclear Translocation Dynamics. Cell systems 11, doi:10.1016/j.cels.2020.08.009 (2020).

590 proteins in living embryos. Dev Cell 56, 2348-2363 e2348, doi:10.1016/j.devcel.2021.07.011 (2021).

14 Dowbaj, A. M. et al. An optogenetic method for interrogating YAP1 and TAZ nuclearcytoplasmic shuttling. J Cell Sci 134, doi:10.1242/jcs.253484 (2021).

15 Viswanathan, R., Hartmann, J., Pallares Cartes, C. \& De Renzis, S. Desensitisation of Notch signalling through dynamic adaptation in the nucleus. EMBO J 40, e107245, doi:10.15252/embj.2020107245 (2021).

16 Grimm, O. \& Wieschaus, E. The Bicoid gradient is shaped independently of nuclei. Development 137, 2857-2862, doi:10.1242/dev.052589 (2010).

17 Liu, F., Morrison, A. H. \& Gregor, T. Dynamic interpretation of maternal inputs by the Drosophila segmentation gene network. Proceedings of the National Academy of Sciences of the United States of America 110, 6724-6729, doi:10.1073/pnas.1220912110 (2013).

18 Ing-Simmons, E. et al. Independence of chromatin conformation and gene regulation during Drosophila dorsoventral patterning. Nature Genetics 53, 487-499, doi:doi:10.1038/s41588-021-00799-x (2021).

19 Gavis, E. R. \& Lehmann, R. Translational regulation of nanos by RNA localization. Nature 369, 315-318, doi:10.1038/369315a0 (1994).

20 Gavis, E. R., Chatterjee, S., Ford, N. R. \& Wolff, L. J. Dispensability of nanos mRNA localization for abdominal patterning but not for germ cell development. Mech Dev 125, 
21 Kinjo, T. et al. FRET-assisted photoactivation of flavoproteins for in vivo two-photon optogenetics. Nature Methods 16, 1029-1036, doi:doi:10.1038/s41592-019-0541-5 (2019).

22 Homans, R. J. et al. Two photon spectroscopy and microscopy of the fluorescent flavoprotein, iLOV. doi:10.1039/C8CP01699B (2018).

23 Garcia, H. G., Tikhonov, M., Lin, A. \& Gregor, T. Quantitative imaging of transcription in living Drosophila embryos links polymerase activity to patterning. Curr Biol 23, 2140-

24 Lucas, T. et al. Live imaging of bicoid-dependent transcription in Drosophila embryos. Curr Biol 23, 2135-2139, doi:10.1016/j.cub.2013.08.053 (2013).

603

25 Bothma, J. P. et al. Enhancer additivity and non-additivity are determined by enhancer strength in the Drosophila embryo. doi:doi:10.7554/eLife.07956 (2015).

26 Syed, S., Wilky, H., Raimundo, J., Lim, B. \& Amodeo, A. A. The nuclear to cytoplasmic ratio directly regulates zygotic transcription in Drosophila through multiple modalities. doi:10.1073/pnas.2010210118 (2021). Alon, U. Network motifs: theory and experimental approaches. Nat Rev Genet 8, 450461, doi:10.1038/nrg2102 (2007). Hoch, M., Seifert, E. \& Jackle, H. Gene expression mediated by cis-acting sequences of the Kruppel gene in response to the Drosophila morphogens bicoid and hunchback. EMBO J 10, 2267-2278 (1991).

613

29 Jacob, Y., Sather, S., Martin, J. R. \& Ollo, R. Analysis of Kruppel control elements reveals that localized expression results from the interaction of multiple subelements. Proc Natl Acad Sci U S A 88, 5912-5916, doi:10.1073/pnas.88.13.5912 (1991).

30 Struhl, G., Johnston, P. \& Lawrence, P. A. Control of Drosophila body pattern by the hunchback morphogen gradient. Cell 69, 237-249, doi:10.1016/0092-8674(92)90405-2 (1992).

31 Tsurumi, A. et al. STAT is an essential activator of the zygotic genome in the early Drosophila embryo. PLoS Genet 7, e1002086, doi:10.1371/journal.pgen.1002086 (2011).

32 Nien, C. Y. et al. Temporal coordination of gene networks by Zelda in the early Drosophila embryo. PLoS Genet 7, e1002339, doi:10.1371/journal.pgen.1002339 (2011).

33 Schulz, C. \& Tautz, D. Autonomous concentration-dependent activation and repression of Kruppel by hunchback in the Drosophila embryo. Development 120, 3043-3049 (1994).

34 Ochoa-Espinosa, A. et al. The role of binding site cluster strength in Bicoid-dependent patterning in Drosophila. Proc Natl Acad Sci U S A 102, 4960-4965, doi:10.1073/pnas.0500373102 (2005). development. Development 111, 601-609 (1991).

36 Huang, A., Rupprecht, J.-F. \& Saunders, T. E. Embryonic geometry underlies phenotypic variation in decanalized conditions. doi:doi:10.7554/eLife.47380 (2020).

37 Kraut, R. \& Levine, M. Mutually repressive interactions between the gap genes giant and Kruppel define middle body regions of the Drosophila embryo. Development 111, 611621 (1991).

38 Rivera-Pomar, R., Lu, X., Perrimon, N., Taubert, H. \& Jackle, H. Activation of posterior 
gap gene expression in the Drosophila blastoderm. Nature 376, 253-256, doi:10.1038/376253a0 (1995).

39 Hulskamp, M., Pfeifle, C. \& Tautz, D. A morphogenetic gradient of hunchback protein organizes the expression of the gap genes Kruppel and knirps in the early Drosophila embryo. Nature 346, 577-580, doi:10.1038/346577a0 (1990).

40 Li, L., Waymack, R., Elabd, M. \& Wunderlich, Z. Two promoters integrate multiple enhancer inputs to drive wild-type $<$ em $>$ knirps $<$ /em $>$ expression in the $<$ em $>$ D. melanogaster $</$ em $>$ embryo. bioRxiv, 2021.2003.2023.436657, doi:10.1101/2021.03.23.436657 (2021).

41 Bothma, J. P. et al. Enhancer additivity and non-additivity are determined by enhancer strength in the Drosophila embryo. Elife 4, doi:10.7554/eLife.07956 (2015).

42 Hoermann, A., Cicin-Sain, D. \& Jaeger, J. A quantitative validated model reveals two phases of transcriptional regulation for the gap gene giant in Drosophila. Developmental Biology, doi:https://doi.org/10.1016/i.ydbio.2016.01.005 (2016).

43 R, K. \& M, L. Mutually repressive interactions between the gap genes giant and Krüppel define middle body regions of the Drosophila embryo. Development (Cambridge, England) 111 (1991).

44 Jaeger, J. et al. Dynamical Analysis of Regulatory Interactions in the Gap Gene System of Drosophila melanogaster. doi:10.1534/genetics.104.027334 (2004).

45 Pankratz, M. J., Busch, M., Hoch, M., Seifert, E. \& Jackle, H. Spatial control of the gap gene knirps in the Drosophila embryo by posterior morphogen system. Science 255, 986989, doi:10.1126/science.1546296 (1992).

46 Niessing, D., Blanke, S. \& Jackle, H. Bicoid associates with the 5'-cap-bound complex of caudal mRNA and represses translation. Genes Dev 16, 2576-2582, doi:10.1101/gad.240002 (2002).

47 Lehming, N. et al. An HMG-like protein that can switch a transcriptional activator to a repressor. Nature 371, 175-179, doi:10.1038/371175a0 (1994).

48 Dubnicoff, T. et al. Conversion of dorsal from an activator to a repressor by the global corepressor Groucho. Genes Dev 11, 2952-2957, doi:10.1101/gad.11.22.2952 (1997).

49 Sako, K. et al. Optogenetic Control of Nodal Signaling Reveals a Temporal Pattern of Nodal Signaling Regulating Cell Fate Specification during Gastrulation. Cell Rep 16, 866-877, doi:10.1016/j.celrep.2016.06.036 (2016).

50 Huang, A., Amourda, C., Zhang, S., Tolwinski, N. S. \& Saunders, T. E. Decoding temporal interpretation of the morphogen Bicoid in the early Drosophila embryo. Elife 6, doi:10.7554/eLife.26258 (2017).

51 Johnson, H. E. et al. The Spatiotemporal Limits of Developmental Erk Signaling. Dev Cell 40, 185-192, doi:10.1016/j.devcel.2016.12.002 (2017).

52 A, H. \& TE, S. A matter of time: Formation and interpretation of the Bicoid morphogen gradient. Current topics in developmental biology 137, doi:10.1016/bs.ctdb.2019.11.016 (2020).

53 Johnson, H. E., Djabrayan, N., Shvartsman, S. Y. \& Toettcher, J. E. Optogenetic rescue of a patterning mutant. Curr Biol, in press (2020).

54 Hannon, C. E., Blythe, S. A. \& Wieschaus, E. F. Concentration dependent chromatin states induced by the bicoid morphogen gradient. doi:doi:10.7554/eLife.28275 (2017).

55 Bischof, J., Maeda, R. K., Hediger, M., Karch, F. \& Basler, K. An optimized transgenesis 
system for Drosophila using germ-line-specific phiC31 integrases. Proc Natl Acad Sci U $S$ A 104, 3312-3317, doi:10.1073/pnas.0611511104 (2007).

68356 Asaoka, M., Sano, H., Obara, Y. \& Kobayashi, S. Maternal Nanos regulates zygotic gene 684 expression in germline progenitors of Drosophila melanogaster. Mech Dev 78, 153-158, 685 doi:10.1016/s0925-4773(98)00164-6 (1998).

68657 Arrizabalaga Muñiz, G. A. Genetic and molecular analysis of the drosophila gene nanos, 687 Massachusetts Institute of Technology, (1999).

68858 Gregor, T. et al. Probing the Limits to Positional Information. Cell 130, 153-164, 689 doi:10.1016/j.cell.2007.05.025 (2007).

$69059 \quad$ Svoboda, K., Denk, W., Kleinfeld, D. \& Tank, D. W. In vivo dendritic calcium dynamics in neocortical pyramidal neurons. Nature 385, 161-165, doi:doi:10.1038/385161a0 (1997).

$69360 \quad$ Pologruto, T. A., Sabatini, B. L. \& Svoboda, K. ScanImage: Flexible software for operating laser scanning microscopes. BioMedical Engineering OnLine 2, 1-9, doi:doi:10.1186/1475-925X-2-13 (2003). 